\title{
Bibliotecas:
}

\section{el largo camino del estado natural a la cooperación}

\author{
Por Concepción Rodríguez-Parada
}

Resumen: A partir de la definición que de la cooperación ofrece la ciencia económica, este trabajo presenta el marco teórico-práctico de la cooperación entre bibliotecas. En primer lugar se presenta una base filosófica, utilizando la teoría del pacto social de Hobbes como modelo explicativo. En segundo, fundamentado en la Biblioteconomía tomada desde una perspectiva conceptual e histórica, se muestra el protagonismo de los bibliotecarios y de las asociaciones profesionales así como las razones económicas o tangibles, e intangibles o de infraestructura que mueven a las bibliotecas a cooperar. La descripción y análisis de experiencias cooperativas en tiempo de crisis muestra la evolución de estas prácticas.

Palabras clave: Pacto social, Cooperación interbibliotecaria, Compartición de recursos, Consorcios, Biblioteca integrada, Biblioteca de uso compartido, Historia.

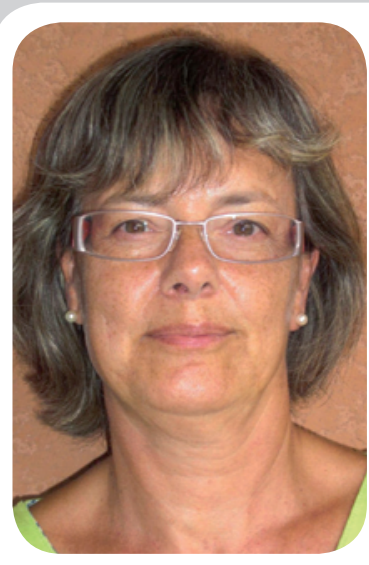

Concepción Rodríguez-Parada es licenciada en filosofía (1982, por la Universitat de Barcelona, UB), diplomada en biblioteconomía y documentación (1987, UB) y doctora en documentación (2009, UB). Desde 1993 es profesora del Departament de Biblioteconomia i Documentació (UB). Miembro de los grupos de investigación Denea (Detecció de necessitats de l'audiència) y Exemplar, y colaboradora del proyecto "Topografía de la espiritualidad femenina mendicante en Cataluña y reinos peninsulares de la Edad Media". Sus líneas de investigación se centran en la gestión de bibliotecas, la deontología, el estudio del patrimonio bibliográfico y la espiritualidad femenina medieval.

\section{Title: Libraries: The long road from their natural state to cooperation}

Abstract: Starting with the definition of cooperation offered by economic sciences, this article presents a theoretical-practical framework of interlibrary cooperation. On the one hand, from a philosophical base it uses Hobbes' theory of the social pact as an explanatory model. On the other, and based in Library Science with a conceptual and historic perspective, it discusses the leading role that librarians and professional associations have assumed, as well as the tangible or economic reasons -and also the intangible factors-that move libraries to cooperate. A description and analysis of cooperative experiences in times of crises show how these practices have evolved.

Keywords: Social pact, Interlibrary cooperation, Resource sharing, Consortia, Joint use of the library, Joint use library, History.

Rodríguez-Parada, Concepción. "Bibliotecas: el largo camino del estado natural a la cooperación". El profesional de la información, 2010, septiembre-octubre, v. 19, n. 5, pp. 457-462.

DOI: $10.3145 /$ epi.2010.sep.02

No queremos la sociedad por sí misma, sino por los honores o los ingresos que pueda reportarnos.

Thomas Hobbes

\section{Introducción}

El objetivo de las líneas que siguen es reflexionar sobre el fenómeno de la cooperación más que mostrar resultados de experiencias concretas. Se trata, pues, de presentar la práctica cooperativa situándola en unas coordenadas económicas, filosóficas y biblioteconómicas, capaces de explicarla de manera global. La economía se utiliza como marco introductorio mientras que la fi- losofía y la biblioteconomía ofrecen el marco teórico y práctico.

Desde la economía se abunda en la idea de que la cooperación define el trabajo conjunto y voluntario de personas o entidades organizadas según unas normas para mejor satisfacer una necesidad común o mejorar su rendimiento. Los beneficios obtenidos se reparten entre dichas personas o entidades, cada una de las cuales recibe un trato igualitario y posee el mismo poder que las demás. En el mismo sentido, el Congreso de Manchester de la Alianza Cooperativa Internacional (ACI, 1995) aprobó una declaración que contenía los 
siete principios básicos del trabajo cooperativo: se trata de una asociación voluntaria; que actúa con independencia; controlada democráticamente por todos sus miembros; los cuales participan en su sostenimiento económico; sus objetivos son el fomento de la educación, la capacitación y la información; interactúa con otras asociaciones similares; y busca mejorar las prestaciones o servicios que ofrece a sus clientes. Esta declaración referida a la cooperación entre empresas es perfectamente aplicable a la establecida entre bibliotecas. Por otra parte, las empresas cooperantes pueden presentarse externamente como comunidad de intereses, consorcio, alianza estratégica, etc., que también están presentes en el mundo de las bibliotecas bajo las denominaciones de redes, sistemas o consorcios.

\section{“Los principios básicos de las empresas cooperativas pueden aplicarse también a la cooperación bibliotecaria"}

\section{Marco teórico-práctico de la cooperación}

\subsection{La filosofía}

Thomas Hobbes (1588-1679) en su obra Leviatán (1651) sostiene que a las personas se les presentan graves obstáculos que no son capaces de superar individualmente. Su capacidad de raciocinio les impele a buscar soluciones que garanticen su seguridad y supervivencia, las cuales sólo son posibles si ceden de forma voluntaria y permanente una parte de sus derechos o libertad a un ente (República, Estado o Leviatán) que los representa a todos y actúa en su nombre. Para que sea efectiva, esta cesión requiere la firma de un contrato o pacto social por parte de todos ellos.

La realidad bibliotecaria permite la extrapolación del principio hobbesiano. Es un lugar común que no hay ninguna biblioteca que, por sí misma, pueda satisfacer las necesidades de información y servicios de sus usuarios en términos de cantidad y calidad. Esta imposibilidad las obliga a abandonar "el estado o condición natural" (acción individual) renunciando con ello al ejercicio de la autonomía en el proceso de toma de decisiones (libertad y poder) en determinados ámbitos de gestión. Dicha renuncia lleva aparejado el establecimiento de un pacto social o contrato para formar redes, sistemas o consorcios. Este acuerdo no es una mera "declaración de intenciones". Es una cesión de derechos voluntaria y expresamente duradera (Boissé, 1994) en la que todos los miembros se comprometen a

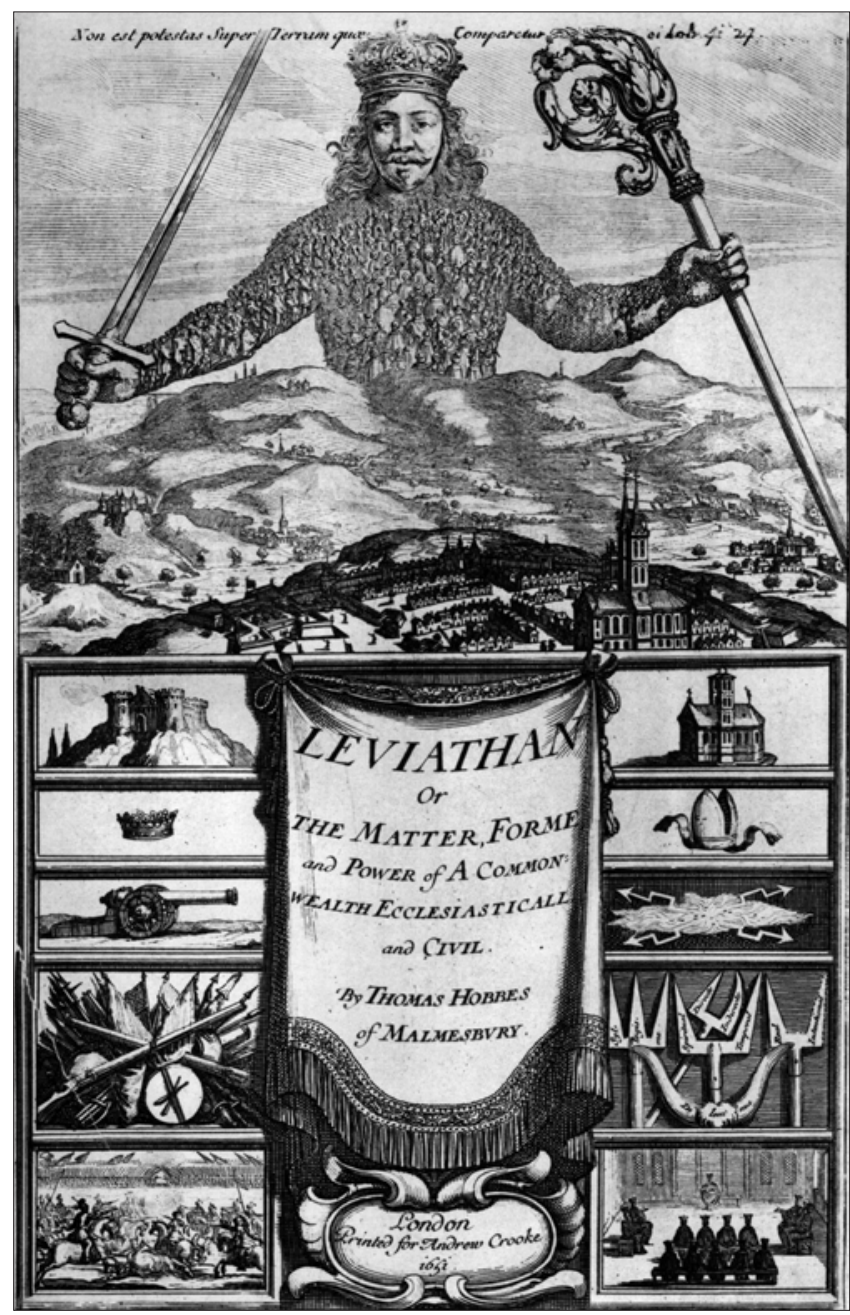

Portada de "Leviathan, or the matter, forme, \& power of a commonwealth ecclesiasticall and civil"

cooperar y trabajar en equipo con la finalidad de alcanzar unos objetivos que reporten beneficios o ganancias tanto a la organización en su conjunto como a cada uno de sus miembros considerados individualmente. Ahora bien, si no contribuye a que la biblioteca sea más eficaz y eficiente entonces no tiene sentido la cooperación.

\subsection{La biblioteconomía}

La biblioteconomía permite una doble aproximación terminológica e histórica. Según la primera, la cooperación engloba el conjunto de actividades realizadas por un grupo de bibliotecas para mejorar servicios y reducir costes mediante un acuerdo firmado por todas ellas que fija las acciones a desarrollar de cara a conseguir objetivos comunes de eficacia, eficiencia y mejorar el propio posicionamiento dentro del mercado de productos y servicios de información. Según Díez-Hoyo (1993) se trata de un proceso de naturaleza contractual y de carácter económico. Sin olvidar este último, por ser la base de cualquier producto, servicio y/o actividad, queremos destacar las dos primeras características. En tanto que procesal (materializada mediante procesos), la actividad cooperativa es susceptible de introducir mejoras que la enriquezcan y la 
hagan más útil a los agentes implicados. Por naturaleza contractual debe entenderse que exige la firma de un convenio escrito con cláusulas consensuadas entre los firmantes sobre los aspectos en los que se quiere cooperar y los recursos necesarios para hacerlo. La práctica cooperativa requiere además una planificación cuidadosa con metas factibles y consensuadas, tiempo, paciencia y una comunicación basada en la confianza entre las partes.

La historia de las bibliotecas ofrece numerosos y excelentes ejemplos de prácticas cooperativas que evolucionan con el paso del tiempo, por lo que pueden distinguirse tres periodos de la cooperación: Antiguo (s. III a. C.- s. XVIII), Medio (s. XIX-1960) y Contemporáneo (1970 en adelante).

\section{"La práctica cooperativa requiere una planificación cuidadosa, tiempo, paciencia y confianza entre las partes"}

\subsubsection{Período antiguo}

Las bibliotecas de Pérgamo y Alejandría (s. III a. C.), a pesar de su reconocida rivalidad, se sabe que hicieron préstamo interbibliotecario (Giordano, 2005).

Durante los siglos XIII-XIV, frailes franciscanos agrupados en las siete custodias que su orden tenía dispersas en Inglaterra y Escocia, elaboraron el Registrum Anglie de libris doctorum et auctorum veterum. Se trata de un catálogo colectivo de manuscritos pertenecientes a bibliotecas monásticas, confeccionado a partir de cuestionarios enviados a 183 bibliotecas, cuyo objetivo principal era conocer la localización precisa de las obras susceptibles de ser copiadas en los scriptoria. Llegó a tener unos 1.400 títulos. Henry de Kirkestede, bibliotecario y luego prior de la abadía Bury St. Edmunds (s. XV) amplió la muestra a 195 bibliotecas, a las que adjudicó un código identificativo, llegando a recoger 674 autores de los que ofrecía algunos datos biográficos para su óptima identificación.

A pesar de la escasez de fuentes, también se tiene noticias de los intercambios de duplicados entre bibliotecas y el intento de elaborar catálogos colectivos durante el Renacimiento.

\subsubsection{Período medio}

Es en el último cuarto del siglo XIX cuando se empieza a gestar el modelo actual de cooperación. Desde 1875 se conocen experiencias cooperativas de préstamo interbibliotecario y de adquisición y catalogación

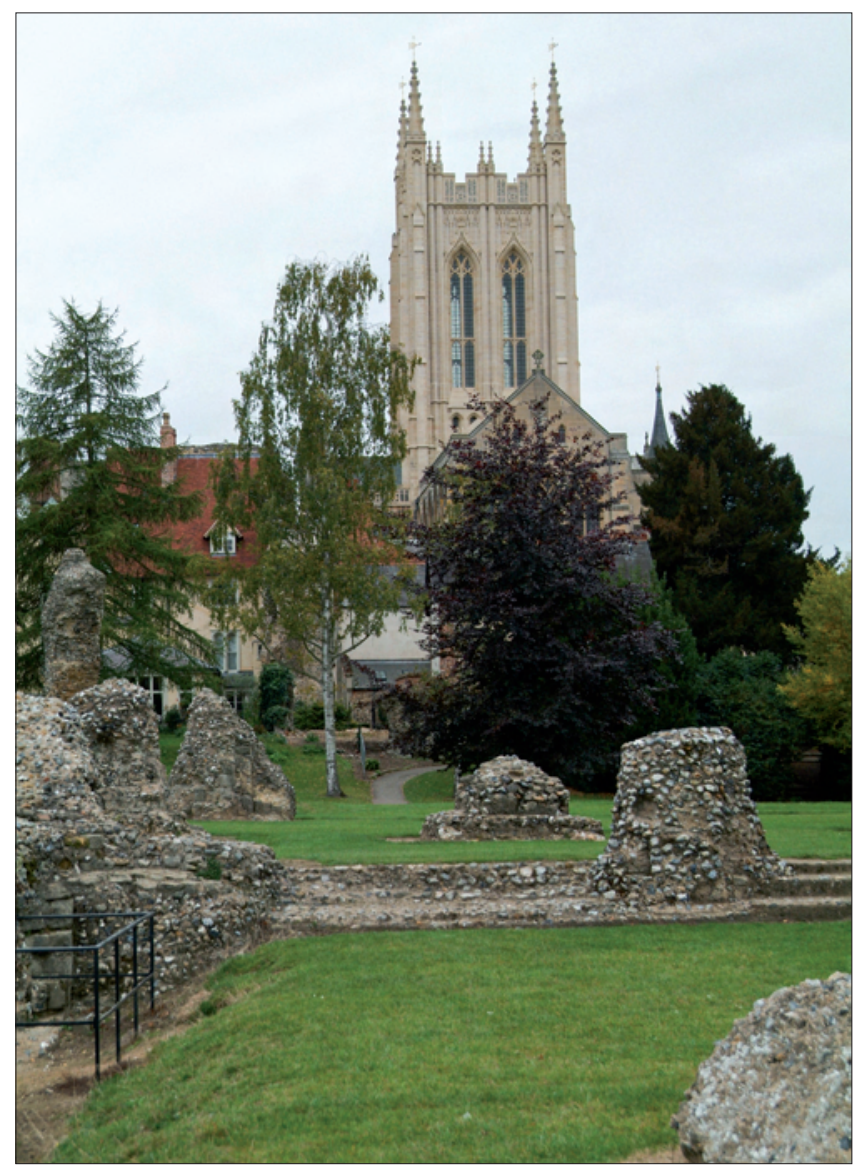

Ruinas de la abadía; al fondo catedral. Bury St. Edmunds, Suffolk, Inglaterra

entre bibliotecas académicas de Canadá y Estados Unidos (Scott, 1976). También se sabe que la State University New York puso en marcha un catálogo colectivo de monografías y revistas de las bibliotecas de colleges. En 1876, Green, uno de los fundadores de la American Library Association (ALA), escribe en el primer número de la revista Library journal sobre la necesidad de establecer acuerdos normativos que regulen el préstamo interbibliotecario, en lo que supone el primer reconocimiento explícito de la exigencia de reglamentar la cooperación.

La influencia de las asociaciones profesionales, principalmente la $A L A$, fue determinante a la hora de fijar el modelo de cooperación bibliotecaria. En su conferencia fundacional celebrada en 1876 en Filadelfia, la $A L A$ definió como uno de sus objetivos "to enable librarians to do their present work more easily and at less expense". En la misma línea, la Charter of 1879 además de promover las prácticas cooperativas interbibliotecarias y reiterar la importancia del factor económico "inducing cooperation in all departaments of bibliothecal science and economy", expresa las ventajas y los valores propios del trabajo cooperativo "promoting [the] library interests [...] by exchanging views, reaching conclusions $[\ldots]$; by disposing the public mind to the founding and improving of libraries; and by cultivating good will among its members". En la con- 
ferencia anual de 1909, el bibliotecario de la Universidad McGill, Charles Gould, señalaba la necesidad de normativizar la cooperación mediante la introducción de "method and order among libraries" y destacaba el papel de los bibliotecarios en este sentido.

La catalogación fue una de las primeras áreas de trabajo puestas en marcha al tratarse de la conditio sine qua non para la elaboración de catálogos colectivos, los cuales, a su vez, son imprescindibles en el préstamo interbibliotecario. El paso siguiente fue la formulación de políticas comunes de desarrollo de colecciones, como, p. ej. el Plan Farmington (Wagner, 2002), elaborado bajo los auspicios de la Association of Research Libraries $(A R L)$ en 1948.

\subsubsection{Período contemporáneo}

La eclosión definitiva de la cooperación tiene lugar a partir de 1970 en Estados Unidos cuando la automatización amplía el campo de trabajo cooperativo: catalogación automatizada, creación de catálogos colectivos, reconversión de catálogos, préstamo interbibliotecario, entre otros. El impacto de las TIC solucionó algunas dificultades no resueltas hasta entonces como facilitar la comunicación y el acceso a la información o garantizar un más rápido acceso a fondos bibliotecarios ajenos, entre otras prestaciones.

El impacto de la edición electrónica sobre la cooperación bibliotecaria es tal que hay quien identifica era digital y cooperación. Los retos que planteaban las publicaciones digitales provocaron que en Norteamérica, desde mediados de la década de 1990, se crearan consorcios de bibliotecas de investigación. Un consorcio es una estructura cooperativa para compartir recursos y reducir gastos, lo cual constituye otra muestra de la preeminencia del factor económico y de la utilidad del pacto social de Hobbes como modelo explicativo.

El término consorcio en un primer momento se asociaba a la adquisición y gestión conjunta de recursos de información electrónica, y era la respuesta de las bibliotecas a la concentración empresarial de la industria editorial (Giordano, 2002, 2005). La creación de consorcios, plataforma desde la que negociar mejor la adquisición conjunta de información electrónica (Térmens, 2007), es vista por las bibliotecas como una ocasión favorable para reducir el gasto por este concepto merced al principio de economía de escala (Sales, 2002). Los consorcios tienen sentido si las bibliotecas o redes integrantes mejoran su situación inicial ya sea porque amplían el número de publicaciones subscritas o porque ahorran en términos absolutos.

No obstante, la tipología y actividades de los consorcios han cambiado con el tiempo. Si al principio aglutinaban exclusivamente a bibliotecas académicas o de investigación, actualmente existen consorcios mixtos de bibliotecas universitarias, públicas y otras, o bien los formados con criterios geográficos. En lo referido al campo de actividades, los consorcios no se limitan a ser un "contrapoder" frente a los distribuidores de información sino que se han desvelado como activos y eficaces agentes de gestión del cambio. En este sentido, la acción consorciada ha permitido que el campo de trabajo cooperativo incluya además de las actividades tradicionales todas las relacionadas con la gestión de la información electrónica: organización de depósitos cooperativos de información electrónica; transferencia de información y materiales; edición de literatura científico-académica; preservación a largo plazo, etc.

\section{Cooperación hoy: respuesta a la crisis}

Si en las líneas precedentes se ha destacado el componente económico como motor del trabajo cooperativo, es innegable que no es ni el primero en el tiempo ni tampoco el único. En la base de la cooperación además de la razón económica de "infraestructura" o material (optimización de los recursos económicos disponibles), pueden distinguirse razones intangibles superestructurales" o "espirituales" (intercambio de recursos para mejorar la calidad del servicio y aumentar la visibilidad de las bibliotecas dentro de la institución y fuera de ella) e inherentes al ejercicio de la profesión. Por ejemplo, lo que movió a los frailes a confeccionar el Registrum Anglie..., no fue el ahorro en sentido estricto sino quizá el afán de poseer, sumado a la vocación de servicio. Ésta última, es, quizá, la motivación subyacente en la cooperación entre bibliotecas y bibliotecarios. Cuando éstos y las asociaciones profesionales fomentan el intercambio de información y experiencias, el fin último es conseguir la mejora del servicio a través del análisis y la resolución de problemas. De algún modo la cooperación no es sino un tipo de actuación a nivel global o universalista avant la lettre. Se trata de una colaboración que en países en vías de desarrollo parte de las bibliotecas para irradiarse y abarcar a otras instituciones y servicios que trabajan en el campo de la salud, la educación y las políticas sociales (Seal, 1998) pero que también puede ampliarse a archivos, museos y otras instituciones culturales. El resultado más evidente para el usuario es el de solicitar a través de una "ventanilla única" todo tipo de información gracias a las ventajas ofrecidas por la red como ya ocurre en Alemania (Kempf, 2008).

En momentos de crisis económica como los actuales en los que según algunos "hacer más con menos no es posible, pero hacerlo de manera diferente con menos sí puede ser", los bibliotecarios tienen la responsabilidad de saber obtener crédito político (Rettig, 2009) mostrando a la sociedad la función democratizadora 


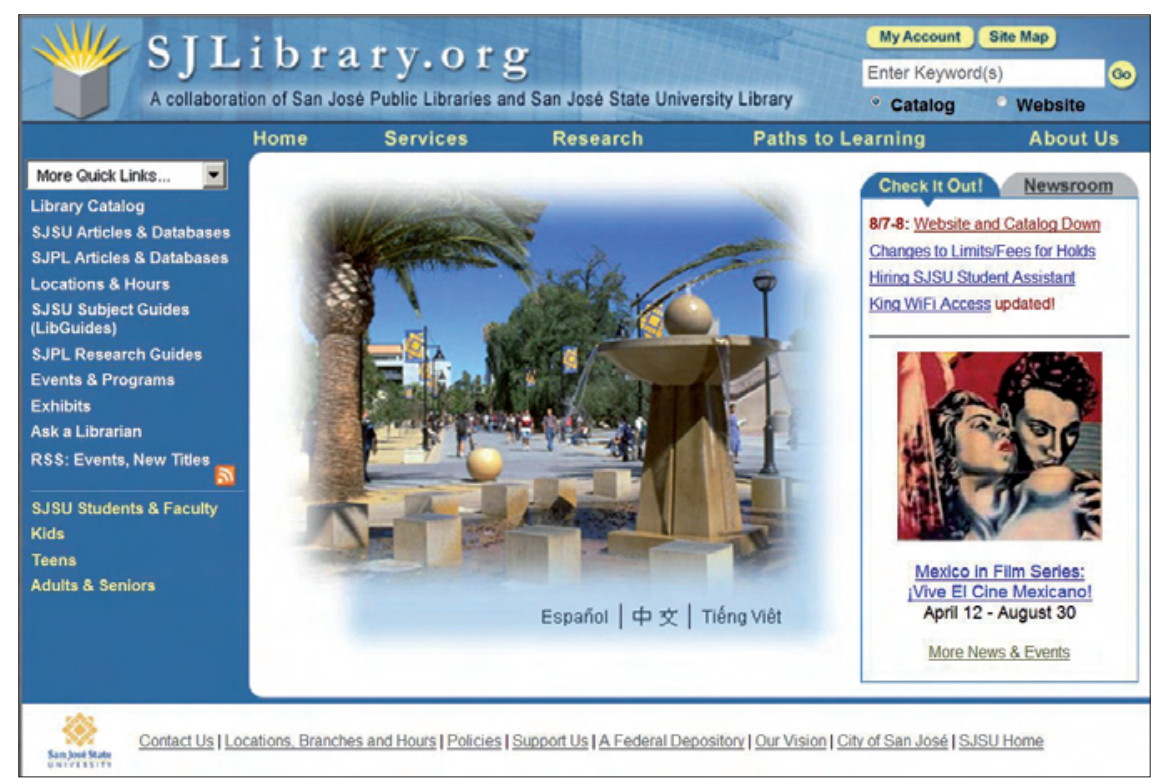

http://www.silibrary.org/

y equitativa que desarrolla la biblioteca a través de su oferta de productos y servicios (Fialkoff, 2010).

Otro tipo de cooperación que se presenta como alternativa a la crisis es el modelo llamado de "biblioteca conjunta" o joint use library. Uno de los primeros y más exitosos ejemplos es la biblioteca $\mathrm{Dr}$. Martin Luther King de San José (California). Sus promotores la definen como un "matrimonio de conveniencia" entre la red de San José Public Libraries y la San José State University. Funciona como biblioteca univeristaria y como biblioteca central del sistema de bibliotecas públicas de la ciudad, dispone de una sola web, el personal trabaja de forma conjunta, y los usuarios tiene un carnet único.

\section{"La vocación de servicio es, quizá, la motivación subyacente en la cooperación entre bibliotecas"}

\section{Conclusiones}

Los motivos que llevan a las bibliotecas a cooperar son de naturaleza racional y utilitarista ya que implican una colaboración entre partes que se necesitan mutuamente para mejorar los servicios al usuario y aumentar su reconocimiento social sin merma de su economía, y esto sólo es posible cuando las bibliotecas comparten recursos. El factor económico es, pues, fundamental pero no el único.

La naturaleza contractual de la actividad cooperativa obliga a las bibliotecas a establecer estructuras orga- nizativas, de coordinación y canales de comunicación eficaces basados en la mutua confianza porque la realidad muestra que no se puede vivir de manera permanente en el estado de naturaleza sino que hay que ceder una parte del propio poder y de la propia libertad -condición del pacto social- para poder vivir en la república de las bibliotecas. Lo que hace falta es acordar cuál es el precio a pagar por ello y las condiciones de pago. Los criterios deben ser equitativos y realistas porque es mejor conseguir lo humanamente posible a lo moralmente deseable (Vernís, 1994). Una vez firmado el acuerdo, hay que perseverar para mantenerlo vigente porque de ello dependen la paz y la seguridad de la república. No obstante, el carácter procesal de la cooperación permite observar que ésta no ha tenido siempre los mismos objetivos, sino que en cada momento se ha adaptado a las necesidades.

\section{Bibliografía}

Alianza Cooperativa Internacional (ACI)

http://www.ica.coop/

http://es.wikipedia.org/wiki/Alianza_Cooperativa_Internacional

http://www.ica.coop/coop/principles.html

ALA. Charter of 1879

http://www.ala.org/ala/aboutala/missionhistory/history/1879/index.cfm

Boissé, Joseph A. "Library cooperation: a remedy but not a panacea". IFLA journal, 1995, v. 21, n. 2, pp. 89-93.

Díez-Hoyo, María-Carmen. "Competir o cooperar: las bibliotecas en los noventa". En: Conferencia de bibliotecarios y documentalistas españoles, 1993, pp. 199-223.

Fialkoff, Francine. "Libraries are a not-so-secret weapon for economic survival”. Library journal.com, 2010

http://www.libraryjournal.com/lj/ljinprint/currentissue/849927-403/ editorial_recession-buster.html.csp

Giordano, Tomasso. "Library consortium models in Europe: a comparative analysis". Alexandria, 2002, v. 14, n. 1, pp. 41-52.

Giordano, Tomasso. "Consorcios bibliotecarios: compartir también criterios y objetivos". El profesional de la información, 2005, v. 14, n. 3, pp 164-165.

http://www.elprofesionaldelainformacion.com/contenidos/2005/mayo/1.pdf

Gómez-Hernández, José-Antonio. "Bibliotecas públicas en tiempos de crisis”. Anuario ThinkEPI, 2010, v. 4, pp. 79-86.

http://www.thinkepi.net/accion-bibliotecas-publicas-tiempos-crisis

Green, Samuel Swett. "Personal relations between librarians and readers". Library journal, v. 1 (October 1876), pp. 74-81.

Hobbes, Thomas. Leviatán, o la materia, forma y poder de una república eclesiástica y civil, 1651.

http://oregonstate.edu/instruct/phl302/texts/hobbes/leviathan-contents.html Comúnmente llamado Leviatán (en inglés Leviathan), es el libro más conocido del filósofo político inglés Thomas Hobbes. El título hace referencia a un monstruo marino descrito en el libro de Job, que suele interpretarse como la representación del demonio. En él Hobbes establece su doctrina de derecho moderno como la base de las sociedades y de los gobiernos legítimos. http://es.wikipedia.org/wiki/Leviatán_(libro) 
Kempf, Klaus. "Unidos somos más fuertes. Cooperación en las bibliotecas científicas alemanas". El profesional de la información, 2008, v. 17, n. 3, pp. 324-330.

Kilgour, Frederick G. The evolution of the book. New York, Oxford University Press, 1998.

http://books.google.es/books?id=Ib_cN9Y9XzOC\&dq=The + Evolution + of + the + Book\&source $=g b s \_n a v l i n k s \_s$

Rettig, Jim. "Library collaboration in the United States". La biblioteca informa al bibliotecario, 2009.

http://www.ucm.es/BUCM/boletin/doc12588.pdf

Rodríguez-Parada, Concepción. "Catalunya o la república de les biblioteques: de l'estat natural a la cooperació”. Item, 2007, n. 46, pp. 7-33.

Sales-Zaguirre, Jordi. Models cooperatius d'assignació de costos en un consorci de biblioteques. ISBN 84-688-1742-2.

http://www.tdx.cat/TDX-0318103-161333

Scott, Edith. "The evolution of bibliographic systems in the United States, 1876-1945". Library trends, v. 25, pp. 293-309.
Seal, Robert A. "Cooperación bibliotecaria: mucho que ganar, poco que perder". En: XXIX Jornadas mexicanas de biblioteconomía, 1998. http://148.226.9.79:8080/dspace/bitstream/123456789/7603/1/mem29 pag41-46.pdf

Térmens-Graells, Miquel. "Los consorcios, una nueva etapa de la cooperación bibliotecaria”. El profesional de la información, 2005, v. 14, n. 3, pp. 166-172.

http://www.elprofesionaldelainformacion.com/contenidos/2005/mayo/2.pdf

Vernís, Alfred. La gestión de las organizaciones no lucrativas. Barcelona: Deusto, 1998. ISBN: 978-84-234-1602-8.

Wagner, Ralph D. A history of the Farmington plan. Lanham, Maryland: The Scarecrow Press, Inc., 2002. ISBN: 0-8108-4359-9.

Concepción Rodríguez-Parada. Universitat de Barcelona, Facultat de Biblioteconomia i Documentació. crodriguezp@ub.edu

\section{ProQuest}
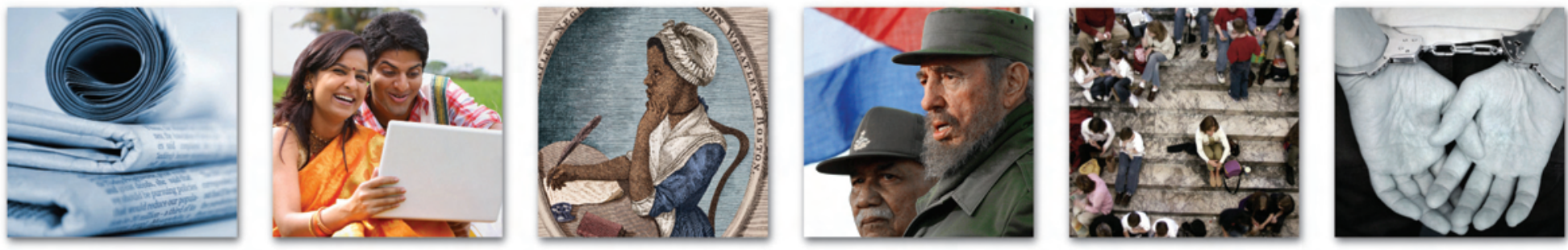

La investigación en Ciencias Sociales busca entender el mundo que nos rodea y cómo los seres humanos estamos conectados, cómo interactuamos, cómo son nuestros entornos sociales, estructuras políticas, razas, géneros...
ProQuest continúa desarrollando y ofreciendo a sus clientes herramientas imprescindibles para conocer y comprender el mundo que nos rodea.

Mejora tu investigación en Ciencias Sociales utilizando International Bibliography of Social Sciences (IBSS) como punto de partida. IBSS, en exclusiva para la plataforma ProQuest, es una de la base de datos más valorada en el campo de la investigación de las ciencias sociales en el mundo y un recurso imprescindible para su biblioteca. 